\title{
(Im)possible Executions of Higher Education Quality Assurance Practices: Imposing Quality Model
}

\author{
Jacob M Selesho \\ Faculty of Management Sciences, Vaal University Technology, \\ Vanderbijipark, South Africa \\ jmselesho@gmail.com
}

\section{Doi:10.5901/mjss.2014.v5n1p507}

\section{Abstract}

This paper reports on the research undertaken at Universities of Technology concentrating on institutionalising the academic quality assurance model for Higher Education Institutions. The paper draw significantly from the impact of quality cycles and its impact on institutions, evolution of quality assurance as well as the preferences in choosing the quality assurance phenomenon. The researcher made use of the descriptive survey as it fits perfectly in this kind of study. Questionnaires and structured interviews were utilised. The purpose of using the qualitative research method is to understand the current quality assurance philosophy exercised by institutions, hence the researcher have decided to use idiographic strategy, in which a single case and its structural coherence with a larger context are examined. The population of this study consists of all six Universities of Technology. Deans of faculties, heads of academic department and programme coordinators the researcher selected only 135 from institutions, as they were able to participate in the study. For the purpose of this study, questionnaire was developed to measure various aspects of quality assurance. From the analysis and interpretation of the empirical findings of this study, it is clear that academics have different perceptions about how the HEQC is conducting its re-accreditation process that flows from internal self-evaluation. It is also proposed that the findings of the self-evaluation process be used to benchmark faculties, and departments at HEls, but also that HE be benchmarked with other quality models worldwide to share best practices. The study concludes that instead of proposing a quality assurance model academics should, rather apply certain principles. Historically, role-players steeped in modern culture have to make it a general practice to build relationships by entering into a dialogue of renegotiating quality principles within the UoT community. Solving problems or contributing positively requires cooperation, and cooperation requires a relationship. The study reveals that quality assurance unit did not have adequate staffing to support academic departments and faculties. These weaken the oversight role played by the quality assurance unit.

Keywords: Framework, Quality Model, Policy,

\section{Introduction}

The role of quality assurance (QA) in a transformed Higher Education (HE) system features prominently in the Report of the National Commission on Higher Education (1996). QA was envisaged by the National Commission on Higher Education (NCHE) as operating within the framework of the South African Qualifications Authority (SAQA) Act 58 of 1995. All these policy documents proposed the development of QA mechanisms for South African higher education institutions (HEls). The policy document that proposed QA in HEls, takes into account the international trends that, to a great extent, informed the task undertaken by institutions in South Africa in building their own QA mechanisms.

Important differences exist among countries and regions of the world with regard to their approach to QA. In addition to numerous national overviews, numbers of studies have attempted to outline the international variations and to systematise the different models used (Verkleij, 2000:90). For the purpose of this study, it is critical that we look at the international standard of self-evaluation in the selected institutions. While, South Africa HEls recognise the fact that internal QA is not simply an internal institutional matter; rather, it is an essential ingredient of an emerging new relationship between government and all HEls to ensure that the procedures and processes followed at institutional level, result in an improvement in quality. At the same time, an institution recognises that its success in ensuring competitiveness and the upholding of standards, together with a greater effectiveness and efficiency, is dependent on the successful implementation of the necessary mechanisms to ensure continuous qualitative improvement through internal evaluation in all its schools and units. 


\section{Statement of the Problem}

HE has always been driven by the need for quality but the explosion of external national quality assurance systems worldwide is making greater demands on institutions to be more transparent in this area. If external accountability has become more systematic, then it is important that an internal procedure becomes more developed and visible to the public, based on the above-mentioned statements universities have used a number of quality model or framework to guide and enforce the culture of quality assurance in different faculties and department. This study intends to ascertain whether there is any framework or model that is fit for purpose at faculties, department and institutional level

\section{Literature Study}

HE has always been driven by the need for quality but the burgeoning of external national QA systems in most countries such as Finland, Denmark and Austria is making greater demands on institutions to be more transparent in this area. By and large, external measures have been useful in promoting quality, although there have been documented cases, here and there, of intrusive procedures (Jensen, 2004:1-3). Nevertheless, if external accountability has become more systematic, then it is important that internal procedures become more developed and transparent to the public.

Internal QA seems, at present, to be receiving a great deal of attention at HEls. These institutions are seeking guidance in determining the most appropriate model on which to base their internal QA policies and procedures. Thus, this study is an attempt to provide information on some of the available models. It is, however, by no means exhaustive. HEls should feel free to experiment, but should take care to avoid unnecessary duplication of effort. Therefore, the best practices in internal self-evaluation are difficult to present.

The requirements of external QA bodies that may be legislated should be met at all times (Jacobs, 2000:69). Many businesses such as industrial and manufacturing models for internal QA are available for adaptation, but HE institutions should decide for themselves which are the most appropriate for their purposes. Such purposes may vary from merely satisfying the external QA agency's requirements, to introducing serious mechanisms at HEls with the purpose of improving internal quality (Woodhouse, 2006:22-24).

According to Du Toit (2001:23-25), Border Technikon (now forming part of the Walter Sisulu University) has adopted an industrial model used by Mercedes Benz South Africa; namely, the South African Excellence Foundation Model (SAEF), for its QA purposes. Du Toit fully agrees with Vroeijenstijn that using an industrial or business enterprise model at HEls is a possibility. The SAEF model is based on the following premise: Customer satisfaction and people satisfaction, impact on society, and suppliers and partnership performance are achieved through leadership driving policy and strategy, customer and market focus, people management, resources and information and processes, leading ultimately to business results (Du Toit, 2001:23-25).

The operations of a HE market demands that students and their families make rational economic choices. The information for making rationale choices, i.e. the measure of the prospective future earnings provided by alternative academic programmes is not available. The fact that students are immature consumers provides for "the implementation of quasi-markets, rather than consumer-orientated markets, for the distribution of academic programmes" (Du Toit, 2001:23-25).

\section{South African Excellence Foundation Model}

Figure 1.1 The South African Excellence Foundation Model with its eleven Criteria (SAEF, 1998:3) 
Source: SAEF, 1998:3

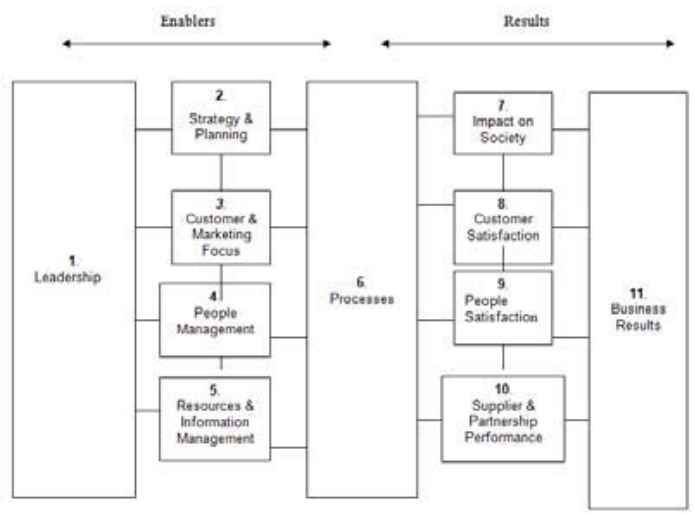

Each of the eleven elements comprising the SAEF model is a criterion that can be used to assess an organisation's progress towards performance excellence.

The terms "enablers" (the first six criteria) and "results" (the last five criteria), are used to group the two main categories of criteria. Before any of the criteria of the SAEF model can be investigated in terms of the assessment of the instructional offering, process-mapping needs to take place so that "steps [can] be studied in terms of time and money review requirements and needs of the customer [students]. Then one must decide what steps in each process add value to your customer [students] and those which do not increase the quality of service to the end-user, should be eliminated" (Du Toit, 2001).

Vroiejenstijn (2001) indicates that in the Netherlands they view industrial models as very difficult to apply at HEls. HEls have developed their own instruments for quality control and quality assessment. The general model applied in HE can be called the External Quality Assessment Model (EQAM). In this model, one may recognise the basic ideas of the accreditation system of the United States (US). The Netherlands has taken the lead in changing the accreditation system and making it comply with the emerging demands of governments, employers and society (Vroiejenstijn, 2001:67).

In designing a quality system, one should keep in mind the following conditions:

- The model should be as simple as possible; It should be cost-effective and stakeholder should trust it.

When applied in the right way, the EQA model is a strong instrument for QA and quality assessment. The main condition for having a powerful instrument is that all stakeholders should trust the system (Vroiejenstijn, 2001:67). A wellfunctioning EQA model will promote quality awareness in the institution.

Figure 1.2: External Quality Assessment Model (EQAM)

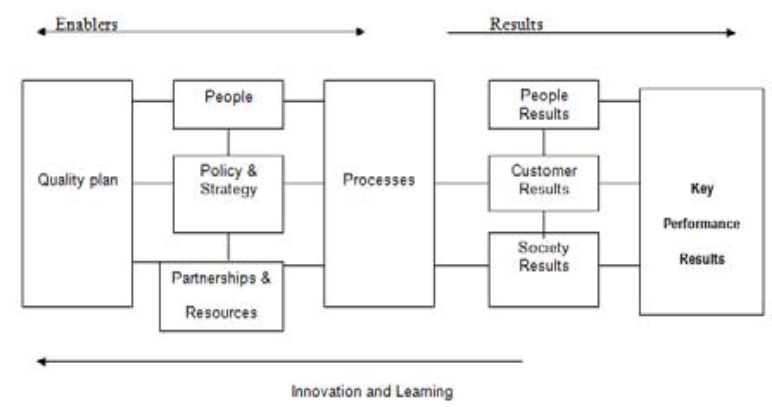

Source: Vroiejenstijn, 2001:67

HE institutions need to establish mechanisms and procedures for self-assessment on an on-going basis where the emphasis is more on accountability at all levels, i.e. academic and support staff, students and management.

QA has thus been proposed as one mechanism of the state for achieving greater efficiency, effectiveness, equity and responsiveness in $\mathrm{HE}$; quality per se is also used to signify a desirable goal for HE itself. The policy discourse 
justifies the restructuring and transformation of $\mathrm{HE}$, and QA by an external state agency, as means of realising these policy goals. In particular, QA is seen as a way of ensuring high quality teaching and learning opportunities for all students; that is, equal educational opportunities for all (Luckett \& Kotecha, 2005:18). QA mechanism procedures should be designed to serve a positive purpose in furthering the interests of the staff and students at HE institutions, and in their teaching and learning (Abbott, 2007:7-10).

\section{Research Methodology}

In this section the methodology used in the study is highlighted and unpacked for the smooth organisation of the process. The researcher made use of the descriptive survey as it fits perfectly this kind of study. The purpose of using the qualitative research method is to describe role of accountability process. Qualitative data were gathered in as many ways as the researcher's creativity permitted (McMillan \& Schumacher, 2007). Although the most widely used sources were observation and interviewing, an analysis of records and documents is common, and it was also used.

The research strategy is idiographic, in which a single case and its structural coherence with a larger context are examined. Cohen, Manion and Morrison (2007:6-9) indicate, that should one favour the views on social reality that stress the importance of the subjective experience of individuals in the creation of the social world, the search for an understanding should focus on different issues and be approached in a totally different way.

\subsection{Population and sample:}

The population of this study consists of all six Universities of Technology (UoTs), academic heads of department (HODs) and quality assurance managers (QAMs). From all deans, heads of department and programme coordinators the researcher selected only 135 from UoT, as they were able to participate in the study.

\subsection{Data collection and Instrumentation:}

Interviews were conducted, supplementary to the administration of questionnaires. Permission for data collection was applied for and obtained from the institutional QA manager. A research instrument is a measuring device used to evaluate more precisely the behaviour being studied. The particular instrument used, depends on the nature of the investigation. Qualitative researchers may use different approaches in collecting data, such as the grounding theory practice, narratology, or storytelling, to mention but a few. The measuring instruments used in this study, were questionnaires and interview sessions.

\section{Results of the Study}

The QA system is intended to support the achievement of the purpose and goal of HE identified in White Paper 3 of 1997. Quality is identified as one of the principles that should guide the transformation of HE, together with equity, effectiveness and efficiency. The White Paper 3 of 1997 identified funding, equity, enrolment planning and QA as key factors for steering the reconstruction and transformation of South African HE and the creation of a HE landscape that is progressively characterised by equitable, high quality and sustainable institutions that are well governed, managed and responsive to the needs of economic growth and social development, and to the consolidation of democracy.

Table 1.1: Quality Assurance Model

\begin{tabular}{|c|c|c|c|c|}
\hline Valid Percent & Frequency & Percent & Valid Percent & Cumulative \\
\hline Yes & 98 & 72.60 & 72.60 & 72.60 \\
\hline No & 37 & 27.40 & 27.40 & 100 \\
\hline Total & 135 & 100 & 100 & \\
\hline
\end{tabular}

The analysis of the submissions indicates that $72.60 \%$ of the respondents were positive that their institution, faculties or department had some sort of framework that guide QA. However, in most cases these frameworks have not yet been translated into plans and strategies. There was not much available documentation, such as manuals or regulations, reflecting QA arrangements. 
Irrespective of how policy-makers within the institution, as well as institutional leaders may decide to shift and place the focus on policy implementation and its discourse and practice; critical questions pertinent to the relevance and academic worth of the institution and its learning programme, will always engage quality scrutiny and enquiry into issues pertaining to the public good.

In order to execute its mandate for quality promotion, the institutional audits and programme accreditation the HEQC need to draw on the expertise, experience and understanding of those who work in the HE sector. While it is true that most HEls are still grappling with the QA issue in a co-ordinated and aligned way, no institution can use this as an excuse for providing inferior standards. It is in this regard that the researcher included the measurement question in the study.

The researcher felt that it was very important to find the viewpoint of the respondents regarding process and approach. It has been mentioned from the outset that the study comprises 135 participants; $93 \%$ believed that quality cannot be measured while $7 \%$ differed from the rest and believe that quality is measurable and can be determined by, among other things, a students' satisfaction survey.

"In our institutions financial incentives, being in the form of grants or other special mechanisms, really assist us in creating support programmes for novice researchers. The university has a plough-back policy, by using the research earnings generated from Master's and Doctoral funding to improve the research capacity as these qualifications are considered as quality indicators."

A large number of respondents agreed with the statement; that it is important to reward institutional quality compliance with funding. 95\% of the respondents seemed to be in support of the idea and it was further revealed that these rewards can be used to enhance the institutional infrastructure subsequent to that to improve the quality of the academic offerings. It must be said that some respondents had a different perception regarding the rewards principle. $5 \%$ of the participants did not fully support the funding mechanisms linked to quality output, saying that the better funding will go to only those elite institutions as they have benefited previously.

\subsection{Analytical summary}

Continuous improvement is the best concept that demonstrates the importance of self-evaluation within institutions of $\mathrm{HE}$. This concept implies that everyone in the institution, individually and collectively, is dedicated and committed to the process of continuous improvement. In order to ensure that the study keeps abreast of trends in terms of articulation and discussions throughout, the impact was measured against the objectives as set out in the study, as well as using continuous monitoring systems. The study paid particular attention to the QA mechanisms, its policy and implementation and process that will assist in proposing a model.

\subsection{Institutional Profiles}

In outlining the institutional profile, the processes were unfolded by means of a sketch, a brief genealogy of the development of the study, drawing on excellent work already done with regard to the profiles of the institution surveyed and referring readers to it for corroboration. It was interesting to note that the four UoTs surveyed represent a total of 145 214 students in the UoTs sector, with more than 22 faculties. The purpose of research of this nature is to contribute to the construction of $\mathrm{HE}$ societies that are able to thrive without a regime of accumulation. To create an open society in $\mathrm{HE}$ is a necessary attribute of constructing a democracy capable of setting rational goals and with the ability to rationally evaluate the means of achieving a sustainable HE system. This cannot be done without loosening the grip of the HE systematic imperatives.

We look forward to the day when the HE community can speak with one voice and say: "We will gladly cooperate with policy makers, government and other stakeholders in meeting human needs, because HE must contribute to skills development. On that day we will be able to say that transformation has been accomplished."

\subsection{The Conceptual Framework}

Current approaches to reform have failed to address the quality transformations necessary to cope with the fundamental problems at a university. The study proposes a viable but undemanding quality model. Who would be against an openminded attitude? Who would be against wanting to see concrete results? The consensus model that the study proposes 
as a quality assurance model is so practicable that every HOD will be able to participate without being highly skilled in quality matters. Academics will not fail to comprehend every step of the model. In attempting to develop a common model of quality assurance that could enhance quality at UoTs, the first approach could be a common vision that academics agree upon. The model ought to encourage academics to do the right thing, in the light of a rational understanding of what the common good requires.

In conclusion, it is a fact that no amount of focus on anomalies will alone transform an institution's quality or its milieu. The model that is suggested will produce transformative knowledge; this model will be a practicable example of the principles set forth in the White paper 3 of 1997 on transforming HE quality principles. It is important to construct a model as an example of productive transformative quality, so that its implementation can be seen to fit into current systems. The model will teach ways to accommodate and assimilate the vast reservoirs of knowledge that already exist in UoTs.

\section{General Discussion}

From the analysis and interpretation of the empirical findings of this study, it is clear that academics have different perceptions about how the HEQC is conducting its re-accreditation process that flows from internal self-evaluation. The self-evaluation process as practised by the different UoTs towards quality improvement is seen not to be performing its intended purpose as some HODs believe that once the self-evaluation process is completed, half the battle is won. This is not the case, as most self-evaluation does not emanate from a critical mindset of robust debate as directed by the department that is being evaluated. The process is merely conducted as an exercise in critique and not applied rigorously; it is simply a process of checking the status quo against a set of criteria.

\section{Conclusion}

All South Africa HE institutions are required to apply for re-accreditation as stipulated by the HEQC. Although most institutions have been using academic self-evaluation as a methodology for many years, very few, if any, have embarked on the journey of the institutional enhancement process supported by a particular quality model or framework. It is thus appropriate to conclude the study by alluding to the fact that self-evaluation is a useful tool for institutional development. It is essential that all support sections at HEls be used as scaffolding mechanisms in the self-evaluation process.

In the quest for modernity, HEls should invest much time in the preparation of self-evaluation and it is important that all staff at HEls be vigilant and confident enough to complain if the system does not work. HODs should help to foster a climate of confidence in the process of self-evaluation and the institution itself, should be continuously scrutinising the strengths and weaknesses of the system in order to keep on improving.

It is also proposed that the findings of the self-evaluation process be used to benchmark faculties, and departments at HEls, but also that $\mathrm{HE}$ be benchmarked with other quality models worldwide to share best practices. Furthermore it is important that a UoT should establish a working structure that will ensure that quality becomes an inclusive process. It is the intention of this study to emphasise that the Quality Unit or Department plays a central and active role in all quality-related matters and not simply a secretarial role.

Instead of proposing a model, the researcher believes that certain principles may be implemented flowing from the working structure and supported by certain propositions. Historically, role-players steeped in modern culture have to make it a general practice to build relationships by entering into a dialogue of renegotiating quality principles within the UoT community.

Solving problems or contributing positively requires cooperation, and cooperation requires a relationship. Therefore, it is important that whatever framework is suggested, it should be coherently focused on strong relationships within the various structures of the UoTs; first the relationship, then the task. Fragmentary and exploitative relationships should be renegotiated at institutional level, with the Quality Unit being responsible for drawing up a relationship document that will guide the departments, faculties, support structures and the institution as a whole. Building consensus and legitimacy implies that the efficacy of self-evaluation is dependent on the contribution and cooperation of all sectors of the institution to connect to one another. In other words, all departments and support structures need to work together consciously and deliberatively for the common good. An indispensable rule of the QA process should be that no department or unit be abandoned if it does not comply. UoTs have a role to play in the consensus building process and in related processes leading to higher quality standards; that is to say in nurturing a more functional and standard legitimacy. 


\section{Recommendations}

The study suggested the following guidelines:

\subsection{Quality Assurance Unit}

There should be proper and adequate staffing in the quality section. This should be a strong team with both quality and quantity being taken care of in order to carry the mandates as tasks to the unit. This is the unit that derive policies and procedures for the QA of an institution. The unit should be in the position to absorb any pressure as a result of institutional territory. In addition to that it is important that such unit must be able to drive the result of any internal or external evaluation. The unit must be capable of assisting the faculty for compliances purposes being improvement plan or audit reports.

\subsection{Faculty Quality Committee}

Faculty Quality Committee: the main purpose of this committee is to ensure that all members of the faculty are conversant with the tenets of quality assurance and are working towards the attainment of the prescribed goals. This committee is accountable to the Institutional quality committee. The committee should be accountable to the institutional QAM and report to the various departments through mandated members. The purpose is to coordinate the activities of QA within the different department of the Faculty.

\subsection{Institutional Quality Committee}

Institutional Quality Committee: this committee may include professionals and members of industries during the internal review process. The external members can be invited when necessary. This committee is responsible to the senate and must give report constantly to the senate on situation of quality at each meeting.

\subsection{Proposed Structure that will guide UoTS dealing with QA}

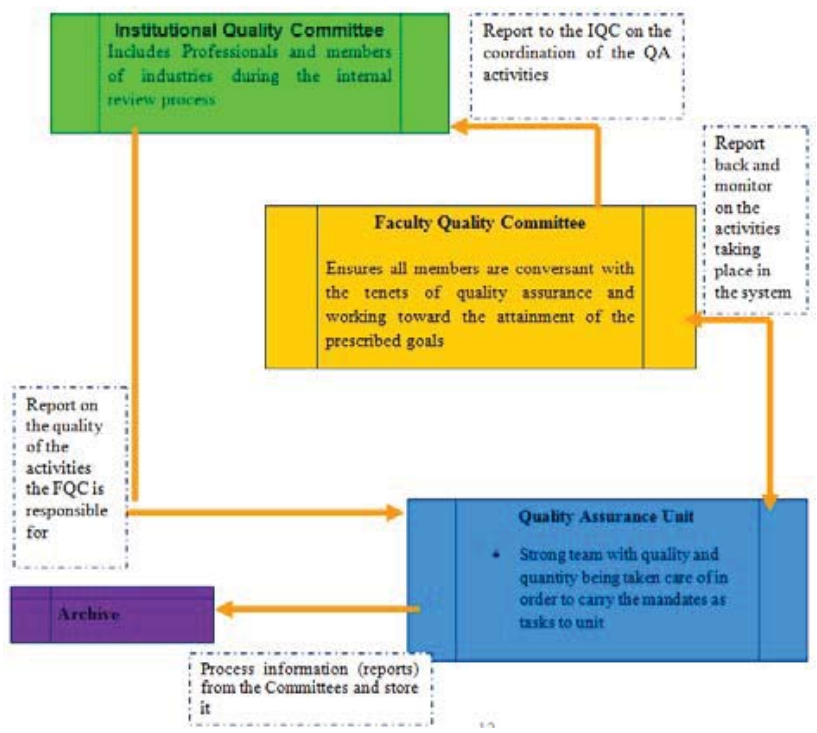

\section{References}

Abbott, $\mathrm{P}$ (2007). QA and Enhancement in Small State: The case of Rwanda, UNESCO, Paris

Cohen, L., Manion, L. and Morrison, K. (2007). Research Methods in Education sixth edition London:Routledge falmer Publishers. 
Council on HE (CHE): (2004). South Africa HE in the First Decade of Democracy. Pretoria: CHE.

De Bruin, K. and Jacobs, G.J. (2000). Interfaculty Perception of Quality Promotion at HE Institution. South African Journal of HE 14(2): 127-129.

Dill, D, (2003). The Catch 22' of Academic Quality: Implication for Universities And Public Policy. North Carolina, Public Policy for Academic Quality, North Carolina, University of North Carolina at Chapel Hill

Du Pre, R. (2000). Committee of Technikon Principals' Report. CTP. Pretoria.

Du Toit, L. (2001). Assessment of Quality in the Management of an Instructional offering at Border Technikon: A System Approach. South African Journal of HE 15 (2): 22-29.

Education White Paper 3 (1997): A Programme for the Transformation of HE, 1997. Pretoria: Department of Education.

Jacobs, D.J. (2000). A Future QA scenario for South African HE. South African Journal of HE, 14(2): 69-74.

Jensen, H.T., (2004). 'Consolidating a quality culture in European universities?' keynote by the Chair, Steering Committee, EUA Institutional Evaluation Programme, Quality Culture Project, Graz Convention, 29-31 May, 2004.

Jongbloed B. (2007). Funding HE: options, trade-offs and dilemmas. Funding Systems and their Effects on HE Public-Private. University of Twentwe Publication

Kasozi, A, (2006). Regulating Transnational HE in Uganda: Consumer Should be Cautious, The Uganda Higher Review, 3, 30-32

Luckett, K. and Kotecha, P. (2005). An analysis of Institutional Profiles: Implications for building a National QA System. Sauvca Occasional Publications and Reports (1).

Maharasoa M.A (2001). Closing the gap: The Relationship between Academic Access Policy and Implementation at Universities in South Africa. Ph.D Dissertation. Bloemfontein: University of the Free State.

McMillan, J.H. and Schumacher, S. (2007). Research in Education. New York: Pearson Education, United States of America.

Republic of South Africa (1995). South African Qualification Authority Act (Act 58 of 1995). Pretoria: Government Printer.

Salmi, J. (2002). Constriction of Knowledge Societies: new challenges for Tertiary Education, Washington DC, Word Bank

South African Excellence Foundation (SAEF) (1998). Criteria for Organization Performance Excellence. Self Assessment, Awards and Prizes.

Verkleij, A.C.L. (2000). Scope and Limitations of Self-Evaluation Process. South African Journal of HE, 14(2): 85-93.

Verkleij, A.C.L. (2001). Different approaches to define research quality. A joint publication between Unisa and Technikon S.A.

Vroeijestijn, T. (1995). Improvement and accountability: Navigating between Scylla and Charybdis. Guide for external quality assessment in HE. London: Jessica Kingsley Publishers.

Vroeijestijn, T. (2001). How to assure quality in HE. A joint publication between Unisa and Technikon S.A.

Woodhouse, D. (2001). QA in HE in 21st century. Launch of the HE Quality Committee. Unpublished.

Woodhouse, D (2006): 'The role of QA Agencies in High Education in the 21st Century', Keynote to 1st International Conference on Assessing Quality in High Education, Lahore, 11-13 December 2006 